\title{
CRITERIA FOR CLOSEDNESS OF VECTOR MEASURES
}

\author{
W. RICKER
}

\begin{abstract}
It is shown that there is a large class of locally convex spaces, which includes, for example, all spaces which are metrizable or Suslin or have the strict Mackey convergence property as well as many dual spaces, with the property that any vector measure assuming its values in such a space is a closed measure.
\end{abstract}

The success of the classical (Lebesgue) integration is based mainly on the fact that the space $L^{1}$ of integrable functions is complete in its natural topology; that is, the topology of uniform convergence of indefinite integrals. Accordingly, it is desirable to have available criteria which guarantee that a vector measure generates a complete $L^{1}$ space. Such vector measures, introduced by I. Kluvánek in [4], are called closed. Their properties are systematically exposed in the monograph $[\mathbf{6}]$; see also $[\mathbf{2}]$.

Let $X$ be a locally convex Hausdorff space. Its dual space will be denoted by $X^{\prime}$. In this note it is shown that the closedness of an $X$-valued measure depends only on the duality between $X$ and $X^{\prime}$. It is also shown that an an $X$-valued measure whose range is metrizable for the relative topology induced by $X$ is a closed measure. This is a slight extension of a result due to Kluvánek [6, IV, Theorem 7.1]. These results are then used to present various sufficient conditions which guarantee that a given vector measure is closed.

1. Preliminaries. An $X$-valued vector measure is a $\sigma$-additive map $m: \mathcal{M} \rightarrow X$ whose domain $\mathcal{M}$ is a $\sigma$-algebra of subsets of a nonempty set $\Omega$. For each $x^{\prime} \in X^{\prime}$, the complex-valued measure $E \mapsto\left\langle m(E), x^{\prime}\right\rangle, E \in \mathcal{M}$, is denoted by $\left\langle m, x^{\prime}\right\rangle$.

If $q$ is a continuous seminorm on $X$, then $B_{q}^{\circ}$ denotes the polar of the closed unit ball, $q^{-1}([0,1])$, of $q$. The $q$-semivariation, $q(m)$, of $m$ is the set function defined by

$$
q(m)(E)=\sup \left\{\left|\left\langle m, x^{\prime}\right\rangle\right|(E) ; x^{\prime} \in B_{q}^{\circ}\right\}, \quad E \in \mathcal{M} .
$$

For each $E \in \mathcal{M}$, the inequalities

$$
\sup \{q(m(F)) ; F \in \mathcal{M}, F \subseteq E\} \leq q(m)(E) \leq 4 \sup \{q(m(F)) ; F \in \mathcal{M}, F \subseteq E\}
$$

hold [6, II, Lemma 1.2].

A complex-valued, $\mathcal{M}$-measurable function $f$ on $\Omega$ is said to be $m$-integrable if it is integrable with respect to every measure $\left\langle m, x^{\prime}\right\rangle, x^{\prime} \in X^{\prime}$, and if, for every

Received by the editors February 8, 1983.

1980 Mathematics Subject Classification. Primary 28B05.

Key words and phrases. Vector measure, closed measure, equicontinuous operator-valued measure. 
$E \in \mathcal{M}$, there exists an element $\int_{E} f d m$ of $X$ such that

$$
\left\langle\int_{E} f d m, x^{\prime}\right\rangle=\int_{E} f d\left\langle m, x^{\prime}\right\rangle
$$

for each $x^{\prime} \in X^{\prime}$. If $f$ is an $m$-integrable function, then the mapping $f m: \mathcal{M} \rightarrow X$ specified by

$$
(f m)(E)=\int_{E} f d m, \quad E \in \mathcal{M},
$$

is called the indefinite integral of the function $f$ with respect to the measure $m$. The Orlicz-Pettis lemma implies that it is a vector measure.

The set of all $m$-integrable functions is denoted by $L(m)$. Members of $\mathcal{M}$ are freely identified with their characteristic function. An $m$-integrable function is said to be $m$-null if its indefinite inttegral is the zero vector measure. Two $m$-integrable functions $f$ and $g$ are $m$-equivalent if $|f-g|$ is $m$-null. The class of all $m$-integrable functions equivalent to a function $f \in L(m)$ is denoted by $[f]_{m}$.

Let $q$ be a continuous seminorm on $X$. If $f$ is an $m$-integrable function, then the $q$-upper integral, $q(m)(f)$, of $f$ is defined by $q(m)(f)=q(f m)(\Omega)$. For any continuous seminorm $q$ on $X$ the relation

$$
q(m)(f)=\sup \left\{\int_{\Omega}|f| d|\langle m, x\rangle| ; x^{\prime} \in B_{q}^{\circ}\right\}, \quad f \in L(m),
$$

is valid [6, II, Lemma 2.2]. Furthermore, the function defined by

$$
f \mapsto q(m)(f), \quad f \in L(m),
$$

is a seminorm on $L(m)$.

Denote by $\tau(m)$ the topology on $L(m)$ which is defined by the family of seminorms (3), for every continuous seminorm $q$ on $X$ or, at least, for enough seminorms $q$ on $X$ to induce the topology on $X$. The resulting locally convex space is not necessarily Hausdorff. The quotient space of $L(m)$ with respect to the subspace of all $m$-null functions is denoted by $L^{1}(m)$.

A set $E \in \mathcal{M}$ is said to be $m$-null if its characteristic function $\chi_{E}$ is $m$-null. Two sets $E, F \in \mathcal{M}$ are $m$-equivalent if their characteristic functions are $m$-equivalent. Since $\left|\chi_{E}-\chi_{F}\right|=\chi_{E} \triangle F$ where $E \triangle F=(E \cup F) \backslash E \cap F$, this is so if and only if $\chi_{E \triangle F}$ is $m$-null. The set of all equivalence classes of $\mathcal{M}$ with respect to $m$-equivalence is denoted by $\mathcal{M}(m)$.

The set $\mathcal{M}(m)$ can be identified with the subset $\left\{\left[\chi_{E}\right]_{m} ; E \in \mathcal{M}\right\}$ of $L^{1}(m)$. Furthermore, as

$$
q(m)(E)=q(m)\left(\chi_{E}\right), \quad E \in \mathcal{M}
$$

for each continuous seminorm $q$ on $X$, the topology and uniform structure $\tau(m)$ has a natural restriction to $\mathcal{M}(m)$ which is again denoted by $\tau(m)$. The topology of $\mathcal{M}(m)$ is given by the family of pseudo-metrics

$$
d_{q}\left([E]_{m},[F]_{m}\right)=q(m)(E \triangle F), \quad E, F \in \mathcal{M},
$$

for enough continuous seminorms $q$ and $X$ to induce the topology on $X$.

A vector measure $m$ is said to be closed $[\mathbf{4}]$ if $\mathcal{M}(m)$ is a complete space with respect to the topology $\tau(m)$. 
Proposition 1. Let $X$ be a locally convex space and $m: \mathcal{M} \rightarrow X$ a vector measure. If range $m(\mathcal{M})=\{m(E) ; E \in \mathcal{M}\}$ of $m$ is metrizable for the relative topology induced by $X$, then $m$ is a closed measure.

ProOF. Choose a sequence $V_{n}, n=1,2, \ldots$, of closed convex balanced neighbourhoods of zero in $X$ with the property that for any zero neighbourhood $V$ in $X$ there exists a positive integer $n$ such that $m(\mathcal{M}) \cap V_{n} \subseteq m(\mathcal{M}) \cap V$; see for example the proof of $[\mathbf{9}$, Theorem 2.1]. Then the topology $\tau(m)$ on $\mathcal{M}(m)$ is given by the sequence of pseudo-metrics

$$
d_{n}\left([E]_{m},[F]_{m}\right)=q(m)(E \triangle F), \quad E, F \in \mathcal{M},
$$

for each $n=1,2, \ldots$, where $q_{n}$ is the gauge functional induced by $V_{n}$. Hence, $\mathcal{M}(m)$ is metrizable.

As $m(\mathcal{M})$ is a bounded set in $X$, it is clear from (1) that there exist constants $\alpha_{n} \geq 1, n=1,2, \ldots$, such that $d_{n}$ is bounded on $\mathcal{M}(m)$ by $\alpha_{n}$, for each $n=1,2, \ldots$. Accordingly, a metric inducing the topology of $\mathcal{M}(m)$ is given by

$$
d\left([E]_{m},[F]_{m}\right)=\sum_{n=1}^{\infty} \alpha_{n}^{-1} 2^{-n} q_{n}(m)(E \triangle F), \quad E, F \in \mathcal{M} .
$$

Let $\left\{\left[E_{r}\right]_{m}\right\}_{r=1}^{\infty}$ be a Cauchy sequence in $\mathcal{M}(m)$. By passing to a subsequence if necessary, we may assume that the sequence $\left\{\left[E_{r}\right]_{m}\right\}_{r=1}^{\infty}$ satisfies the inequalities

$$
d\left(\left[E_{r}\right]_{m},\left[E_{r+1}\right]_{m}\right) \leq 2^{-r}, \quad r=1,2, \ldots
$$

Let $E=\limsup E_{r}$. The proof is completed by showing that $[E]_{m}$ is the limit of the sequence $\left[E_{r}\right]_{m}, r=1,2, \ldots$, for which it suffices to show, by $(5)$, that

$$
d\left([E]_{m},\left[E_{r}\right]_{m}\right) \leq \sum_{s=r}^{\infty} d\left(\left[E_{s}\right],\left[E_{s+1}\right]_{m}\right)
$$

for each $r=1,2, \ldots$ This inequality follows from the countable subadditivity of semivariation, the formula (4) and the inclusion $E \triangle E_{r} \subseteq \bigcup_{s-r}^{\infty} E_{s} \triangle E_{s+1}$, valid for each $r=1,2, \ldots$.

If $\rho$ is any locally convex topology on $X$, then $X_{\rho}$ will denote the space $X$ equipped with the topology $\rho$. Let $m: \mathcal{M} \rightarrow X$ be a vector measure. If $\rho$ is a locally convex topology on $X$ consistent with the duality between $X$ and $X^{\prime}$, then the Orlicz-Pettis lemma implies that the set function $m_{\rho}: \mathcal{M} \rightarrow X_{\rho}$ given by $m_{\rho}(E)=$ $m(E), E \in \mathcal{M}$, is also a vector measure. We will say that $m$ is closed for the topology $\rho$ if $m_{\rho}$ is a closed measure.

PROPOSITION 2. Let $X$ be a locally convex space and $m: \mathcal{M} \rightarrow X$ a vector measure. Then $m$ is a closed measure if and only if $m$ is a closed measure for every locally convex topology on $X$ consistent with the duality between $X$ and $X^{\prime}$.

ProOF. Let $\sigma$ denote the weak topology, $\sigma\left(X, X^{\prime}\right)$, on $X$. It suffices to show that a closed measure $m: \mathcal{M} \rightarrow X$ induces a closed measure $m_{\sigma}: \mathcal{M} \rightarrow X_{\sigma}$ and secondly that if a measure $m: \mathcal{M} \rightarrow X$ is closed for the weak topology on $X$, then $m$ is also closed for any locally convex topology on $X$ consistent with the duality between $X$ and $X^{\prime}$. 
Suppose then that $m$ is closed. There exists a localizable measure $\lambda$ on $\mathcal{M}$ such that the measure $\left\langle m, x^{\prime}\right\rangle$ is absolutely continuous with respect to $\lambda$ for each $x^{\prime} \in X^{\prime}$ [5, Corollary 13]. Since $X_{\sigma}$ has the same dual space as $X$, it follows from [6, IV, Theorem 7.3] applied to the space $X_{\sigma}$ that $m$ is closed for the weak topology on $X$.

Now suppose that $m_{\sigma}$ is closed. Let $\rho$ be a locally convex topology on $X$ consistent with the duality between $X$ and $X^{\prime}$. Clearly $L^{1}\left(m_{\rho}\right)$ and $L^{1}\left(m_{\sigma}\right)$ are equal as vector spaces; denote this space simply by $L^{1}$. Since the seminorms determining the topology of $X_{\sigma}$ are a subfamily of those which determine the topology of $X_{\rho}$, it is clear that $\tau\left(m_{\sigma}\right)$ induces a weaker topology on $L^{1}$ than $\tau\left(m_{\rho}\right)$. If $q$ is a continuous seminorm on $X_{\rho}$, then it follows from (2) that

$$
\left\{f \in L^{1} ; q\left(m_{\rho}\right)(f) \leq 1\right\}=\bigcap_{x^{\prime} \in B_{q}^{\circ}}\left\{f \in L^{1} ; \int_{\Omega}|f| d\left|\left\langle m, x^{\prime}\right\rangle\right| \leq 1\right\}
$$

is valid. Hence, $L^{1}$ has a base of neighbourhoods of zero with respect to $\tau\left(m_{\rho}\right)$ which are $\tau\left(m_{\sigma}\right)$-closed. It follows that if a $\tau\left(m_{\rho}\right)$ Cauchy net $\left\{f_{\alpha}\right\}_{\alpha \in A}$ has a $\tau\left(m_{\sigma}\right)$ limit $f$, then also $f=\lim _{\alpha} f_{\alpha}$ with respect to $\tau\left(m_{\rho}\right)$; see, for example, $[\mathbf{7}, \S 18$, Proposition 4.4]. This observation together with the fact that $\mathcal{M}(m)$ is identified with the subset $\left\{\left[\chi_{E}\right]_{m} ; E \in \mathcal{M}\right\}$ of $L^{1}$ implies that $\mathcal{M}\left(m_{\rho}\right)$ is complete.

2. Sufficient conditions for closedness. It is well known that the range of a vector measure with values in a quasicomplete space is a relatively weakly compact set. Furthermore, a large class of locally convex spaces have the property that weakly compact subsets are metrizable for the weak topology. These two observations together with the results of $\S 1$ provide an effective means for verifying the closedness of a large class of vector measures. Recall that a locally convex Hausdorff space is Suslin if it is the continuous image of a Polish space [1, Chapter $9, \S 6.2]$.

Proposition 3. Let $X$ be a quasicomplete locally convex space and $m: \mathcal{M} \rightarrow X$ a vector measure. Each of the following conditions is sufficient to guarantee that $m$ is a closed measure.

(i) $X$ is metrizable.

(ii) $X$ has the strict Mackey convergence property.

(iii) $X$ is a Suslin space.

(iv) $X^{\prime}$ is weak-* separable.

Proof. (i) is immediate from Proposition 1; see also [6, IV, Theorem 7.1].

(ii) Such spaces have the property that bounded sets are metrizable for the relative topology; see, for example, $[\mathbf{9}]$ and the references therein. The result then follows from Proposition 1.

(iii) Let $\overline{\mathrm{bco}} m(\mathcal{M})$ denote the closed convex balanced hull of the range of $m$. Since $X$ is Suslin for the weak topology $[\mathbf{1 0}]$ and $\overline{\mathrm{bco}} m(\mathcal{M})$ is weakly compact, it follows that $\overline{\mathrm{bco}} m(\mathcal{M})$ is Suslin and compact in $X_{\sigma}$ and hence, is metrizable (for the weak topology) [1, Chapitre 9, Appendice 1, Corollaire 2 to Proposition 3]. Propositions 1 and 2 then imply the $m$ is closed.

(iv) Since $\overline{\mathrm{bco}} m(\mathcal{M})$ is weakly compact it is metrizable for the weak topology $[\mathbf{3}$, Proposition 3.2]. Again Propositions 1 and 2 imply that $m$ is closed. 
PROPOSITION 4. Let $X$ be a locally convex space and $X^{\prime}$ be equipped with some locally convex topology. Each of the following conditions is sufficient to guarantee that a vector measure $m: \mathcal{M} \rightarrow X^{\prime}$ is closed.

(i) $X$ is separable and barrelled and the topology on $X^{\prime}$ is consistent with the duality between $X$ and $X^{\prime}$.

(ii) $X$ is separable and bornological and $X^{\prime}$ has a quasicomplete topology which is consistent with the duality between $X$ and $X^{\prime}$.

(iii) $X$ is quasicomplete, barrelled and nuclear and $X^{\prime}$ has the strong dual topology.

Proof. (i) Since $\overline{\mathrm{bco}} m(\mathcal{M})$ is $\sigma\left(X^{\prime}, X\right)$ bounded it follows that $\overline{\mathrm{bco}} m(\mathcal{M})$ is equicontinuous in $X^{\prime}$ and hence, is metrizable for $\sigma\left(X^{\prime}, X\right)$; see, for example, [7, $\S 21$, Proposition 3.4]. Propositions 1 and 2 then imply the result.

(ii) As $X$ is bornological and $\overline{\mathrm{bco}} m(\mathcal{M})$ is balanced, convex and $\sigma\left(X^{\prime}, X\right)$ compact, it follows that $\overline{\mathrm{bco}} m(\mathcal{M})$ is an equicontinuous part of $X^{\prime}$. The proof can then be completed as in (i).

(iii) Since $\overline{\mathrm{bco}} m(\mathcal{M})$ is a bounded set in $X_{\beta}^{\prime}$ and $X$ is a subspace of the dual of $X_{\beta}^{\prime}$, it follows that $\overline{\mathrm{bco}} m(\mathcal{M})$ is $\sigma\left(X^{\prime}, X\right)$ bounded. Accordingly, $\overline{\mathrm{bco}} m(\mathcal{M})$ is an equicontinuous part of $X^{\prime}$. It follows that $\overline{\mathrm{bco}} m(\mathcal{M})$ is metrizable (and compact) for the relative topology induced by $X_{\beta}^{\prime}[\mathbf{1 1}$, Proposition 50.2]. Proposition 1 then implies that $m$ is closed.

In $[8]$, the theory of integration with respect to closed spectral measures was used to obtain results in operator theory. Applications of this kind make it desirable to have available criteria for the closedness of operator-valued measures.

Let $X$ and $Y$ be locally convex spaces. The space of all continuous linear operators from $X$ into $Y$, equipped with the topology of pointwise convergence, is denoted by $L(X, Y)$.

An $L(X, Y)$-valued measure is said to be equicontinuous if its range is an equicontinuous part of $L(X, Y)$.

Proposition 5. Let the space $X$ be separable and the space $Y$ be metrizable. Then any equicontinuous $L(X, Y)$-valued measure is closed.

In particular, if $X$ is a separable Fréchet space, then any $L(X, X)$-valued measure is closed.

ProOF. The proof follows from Proposition 1 since equicontinuous subsets of $L(X, Y)$ are metrizable for the relative topology induced by $L(X, Y)$; see $[\mathbf{1 1}$, Exercise 32.8 ].

The second statement follows from the fact that if $X$ is a Fréchet space, then any $L(X, X)$-valued measure is equicontinuous. The statement also follows from Proposition 3(iii) since $L(X, X)$ is a Suslin space $[\mathbf{1 0}]$.

The author wishes to thank Professors I. Kluvánek and S. Okada for valuable discussions.

\section{REFERENCES}

1. N. Bourbaki, Topologie générale. II (Nouvelle Édition), Chapitres 5 à 10, Hermann, Paris, 1974. 
2. C. H. Brook and W. H. Graves, Closed measures (Proc. Conf. Integration, Topology and Geometry in Linear Spaces), Contemporary Math., vol. 2, Amer. Math. Soc., Providence, R.I., 1980, pp. 145-160.

3. K. Floret, Weakly compact sets, Lecture Notes in Math., Vol. 801, Springer-Verlag, Berlin and New York, 1980.

4. I. Kluvánek, The range of a vector valued measure, Math. Systems Theory 7 (1973), 44-54.

5. __ Conical measures and vector measures, Ann. Inst. Fourier (Grenoble) 27 (1977), 83-105.

6. I. Kluvánek and G. Knowles, Vector measures and control systems, North-Holland, Amsterdam, 1976.

7. G. Köthe, Topological vector spaces. I, Die Grundlehren der Math. Wissenschaften, No. 159, Springer-Verlag, Berlin, 1969.

8. W. Ricker, On Boolean algebras of projections and scalar-type spectral operators, Proc. Amer. Math. Soc. 87 (1983), 73-77.

9. E. Saab, On the Radon-Nikodym property in a class of locally convex spaces, Pacific J. Math. 75 (1978), 281-291.

10. G. E. F. Thomas, Integration of functions with values in locally convex Suslin spaces, Trans. Amer. Math. Soc. 212 (1975), 61-81.

11. F. Treves, Topological vector spaces, distributions and kernels, Academic Press, New York, 1967.

SChool of Mathematical Sciences, The Flinders University of South AusTrAlian, BEDFORd PARK 5042, AUstralia 